\title{
LA CONTAMINACIÓN AUDITIVA: PRÁCTICAS COTIDIANAS QUE NATURALIZAN EL RUIDO COMO FORMA DE COMUNICACIÓN EN NIÑOS DE BÁSICA PRIMARIA
}

\author{
RUTH MARITHZA HIGUERA IBÁÑEZ \\ Psicóloga \\ Especialista en Necesidades Educativas Especiales \\ Estudiante del Doctorado en Ciencias Sociales, Niñez y Juventud \\ Universidad de Manizales- CINDE \\ ruthmarithza@gmail.com
}

\section{Cómo citar este artículo:}

Higuera, R. (2011). La contaminación auditiva: prácticas cotidianas que naturalizan el ruido como forma de comunicación en niños de Básica Primaria. Espiral, Revista de docencia e investigación. 1 (1). 45 - 52

\section{Resumen}

El presente artículo plantea algunos aspectos que han naturalizado el ruido como parte de la comunicación en el aula tanto entre estudiantes como en la díada docente - estudiante. Para ello, se refexiona alrededor de una actividad programada en la asignatura de Ciencias Naturales con estudiantes de quinto grado de básica primaria del Colegio Roberto García Peña, sede B jornada de la tarde en el 2010 y 2011 acerca de la Contaminación Ambiental que puede identifcarse en la sede. Parte de los hallazgos remiten a la invisibilidad del ruido como contaminante potencial dentro y fuera del aula, además de ser visto como una característica normal en el comportamiento de los estudiantes por su condición misma de ser niños y niñas.

Palabras clave: Contaminación auditiva, ruido, comunicación, naturalización, comportamiento.

\footnotetext{
Abstract

This article aims to raise some issues that have naturalized noise as part of the communication in the classroom both between the dyad students and teachers. In order to do this, we analyze a a scheduled activity in the subject of Natural Sciences with ffth grade students of primary school, Roberto Garcia Peña, campus B, 2010 and 2011, afternoon shift, about the environmental pollution that can be identifed at the campus. Some of the fndings refer to the invisibility of noise as a potential contaminant inside and outsi-
}

de the classroom, as well as being seen as a normal feature in the performance of students by their very condition of being children.

Key words: Noise pollution, noise, communication, naturalization, behavior.

\section{Introducción}

Es muy común plantear factores determinantes de contaminación y entre ellos ubicar aquellos que se relacionan con la contaminación acústica. Sin embargo, cuando se hace el ejercicio refexivo de observar las aulas de clase o incluso preguntarse por lo que pasa en una institución educativa, es inevitable hacerlo alrededor de cómo no vemos el tono de voz, que al hablar en ciertas condiciones con otros, junto con las actividades que se plantean como parte del proceso mismo de enseñanza - aprendizaje, empieza a convertirse en factores que generan ruido y que, igualmente, pueden perturbar $\mathrm{u}$ obstaculizar el desarrollo adecuado del acto de aprender, al afectar el clima de aprendizaje.

Por ello, cabe preguntarse: ¿En qué condiciones el ruido se convierte en un aspecto naturalizado de nuestra forma de relacionarnos en 
el aula? Se parte del concepto de ruido como aquello, que además de ser o constituirse en un sonido molesto, genera efectos fsiológicos y psicológicos nocivos para una o más personas que comparten un mismo escenario y que, por ende, puede ser visto como un contaminante auditivo (Flores-Domínguez, 2009).

El ser contaminante, se relaciona directamente con la perturbación que puede generarse en las distintas actividades que surgen en los grupos, que interfere directamente la comuni-

cación hablada, as- El ser contaminanpecto fundamental para la convivencia, máxime, cuando es en el aula, pero que no sólo se queda en este ámbito de lo relacional, sino que a nivel individual, puede perturbar el sueño, el descanso, la relajación, impedir la concentración y el aprendizaje, para propiciar este, se relaciona directamente con la perturbación que purede generarse en las distintas actividades que suirgen en los grupos, que interfere directamente la comunicación hablada tados de cansancio y tensión que, incluso, pueden conllevar a enfermedades de tipo nervioso y cardiovascular (Frers, 2009).

En este artículo se presenta algunos aspectos que han llevado a invisibilizar el ruido como factor contaminante en la interacción que surge dentro y fuera del aula, y donde el docente lo ha caracterizado como parte de la naturaleza comunicativa en sus estudiantes e, incluso, como parte de su forma de relacionarse con ellos.

\section{Oír sin entender}

Parte de la afección que ocasiona el ruido es invisible cuando se conversa con otro ya que no se logra dimensionar conscientemente los mecanismos involucrados en este proceso. Sólo se empieza a darle importancia cuando se tiene algún tipo de complicación auditiva, por ejemplo, cuando no se oye el timbre del teléfono, cuando se difculta comprender programas en la televisión, o incluso, cuando no se logra tener claridad de las palabras que pronuncia otra persona, además de empezar a hablar con un tono más alto de lo usual. Podemos oír pero no entender lo que escuchamos, con lo cual se puede estar ante el inicio de la sordera y la aparición de la fatiga auditiva como lo expresa Freirs (2009).

No es sólo el problema de no comprender lo que se escucha o incluso de oír, sino todo aquello que puede estar asociado a su alrededor, como la falta de equilibrio, la irritabilidad, la ansiedad, las alteraciones del ritmo cardiaco, la falta de concentración y el desorden del sueño que trae consigo la predisposición a padecer trastornos psiquiátricos.

Por supuesto, hablar del ruido, nos encausa a hablar de la experiencia subjetiva del mismo, puesto que un mismo ruido puede indisponer a unos y ser indiferente para otros. Sin embargo, puede ser que escudados en ese aspecto subjetivo simplemente se toma como un proceso natural la presencia misma del ruido (Freirs, 2009).

En cuántas ocasiones sentimos estrés, o impotencia cuando no logramos concentrarnos en la actividad que se realiza o simplemente, cuando de manera repentina experimentamos una pérdida de energía frente a las tareas que se realizan; por ello, al momento de hacerlo se estamos lleno de tantos sonidos que, se presta más atención a la conversación de otro, a las actividades que realizan los demás o incluso, a sonidos que están fuera del escenario donde se realiza dicha tarea, o se podría simplemente estar en una actividad lúdica - recreativa donde todos gritan por la emoción de lo que pasa en la actividad, pero, en la cual es más difícil 
lograr que los participantes sepan a ciencia cierta lo que deben y se espera que realicen.

Se podría estar frente a la contaminación acústica, ser parte de ella, sentirse enfermos, agotados y así preguntarse sobre el por qué se experimenta esas sensaciones que enferman no sólo la actitud y la aptitud sino tam-
Se podría estar frente a la contaminación acústica, ser parte de ella, sentirse enfermos, agotados y así preguntarse sobre el por qué se experimenta esas sensaciones que enferman no sólo la actitud y la aptitud sino también. el cuerpo bién el cuerpo, que en la actualidad puede considerarse como la única forma de estar en el mundo y de comunicarse con él.

\section{La escuela escenario para el ruido}

Al ser la escuela un espacio que construye y deconstruye las formas sociales, es el escenario de predilección donde se conjuga la interacción de aspectos normativos, organizativos y de signifcación, aspecto último que conlleva de la mano valores que se comparten y orientan las prácticas educativas, pero que, también incluyen experiencias que pueden afectar la salud de todos los agentes involucrados en el acto educativo. Motivo por el cual, las generalizaciones en este escenario no tendrían lugar debido a) la diferencia que existe frente a cada una de las características de los maestros como personas, b) las habilidades cognitivas, c) las habilidades sociales, d) las habilidades docentes (didáctica y pedagogía), y e) el área de desempeño (básica primaria, básica secundaria y superior), aspectos que se conjugan a través del lenguaje en todas sus formas.

Al tratarse de los agentes que promueven directamente el aprendizaje, es decir, de los docentes, la predilección del trato sujeto a sujeto implica una interacción basada en el lenguaje en todo su sentido, pero donde las palabras confguradas en el habla, son el instrumento de base para el aprendizaje que da inicio a la transformación de la información en conocimiento en el ámbito del aula.

Esa voz del maestro acentúa su importancia, junto con todo aquello que experimenta y que de una manera $u$ otra repercute en la interacción en el aula. Por ello, las alteraciones de la salud mental de los educadores han sido abordadas según Del Valle y Almirall (2008) por diferentes autores como Travers y Cooper, quienes en su estudio acerca de la salud mental, la satisfacción en el trabajo y el estrés en la ocupación de los docentes expresan que el tiempo de dedicación es relevante puesto que según éste, se reporta más estrés y síntomas psicosomáticos frente a aquellos que tienen menor tiempo de dedicación.

Por otra parte, Messing y Colaboradores identifcan en 14 docentes como estresores laborales la secuencia de eventos, la corta duración de la dirección de la mirada en un mismo punto en el aula, la simultaneidad de actividades, las condiciones poco confortables de temperatura y humedad ambientales en las aulas.

Y Pomenta cita varios factores relacionados con las frustraciones y desajustes en el estado de salud mental de los educadores, la necesidad de mantener las explicaciones a un nivel coherente y comprensible, estimular el interés y atención de los alumnos y mantener la disciplina.

$\mathrm{Al}$ detenerse en estos aspectos planteados por los anteriores autores (citados en Del Valle y Almirall, 2008) se puede observar la necesidad de un clima educativo que favorezca la comunicación y comprensión en el aula de los distintos contenidos, pero donde el ruido podría ser ese factor que obstaculice 
el desarrollo normal de los procesos cognitivos que interactúan en el acto de aprender que afectan el nivel de atención requerido para aprehender el conocimiento e incluso facilitar las modifcaciones en el comportamiento en los estudiantes en cuanto al cómo se comunican entre ellos, con el docente y la actividad académica misma.

Si se presenta una modifcación de comportamiento del tipo donde los estudiantes hablen de manera continua, corran por el salón de clases o incluso lleguen a agredirse física y / o verbalmente, el docente sentirá además de estrés, la necesidad de subir su tono de voz y si con ello, se le agrega una continuidad no sólo durante la jornada en que se encuentre, sino del día a día, se tendrá la posibilidad de una patología en su voz, uno de los problemas de salud que más afecta a los docentes y que repercute directamente sobre su labor profesional, pero que además abonará al tipo de dinámica comunicativo- relacional que se da en el aula.

Por ejemplo, Calas y Colaboradores en un estudio realizado en Francia con 100 docentes, encontraron que la consulta frecuente era alrededor de: a) disfonía con modifcación de la voz hablada, b) fatiga vocal, c) falta de resistencia, d) dolor y sensación anormal (picazón, quemadura, garganta seca) y, e) afonía (Citado en Del Valle y Almirall, 2008).

Para Preciado López (2009), la patología vocal es más frecuente en docentes de niveles inferiores de la educación, sobretodo en educación infantil y primaria, debido en parte al mayor número de horas de trabajo y a la cantidad de estu- diantes que tienen en el aula. Además que la edad de los estudiantes está relacionada con la existencia de un nivel superior de ruidos en la clase, lo cual les obliga a forzar la voz para hacerse oír.

A ello se suma que en estos niveles es frecuente que las maestras pasen de la voz hablada a la cantada e imiten sus voces en frecuencias superiores a la propia, lo cual debería incluir por el tipo de trabajo que las horas laborales contemplen períodos signifcativos de reposo vocal entre las clases y el número de estudiantes no sobrepase de 25 niños (primaria y secundaria) o 20 niños si es en preescolar, pero que estos aspectos no lo contempla la política educativa en Colombia, que está más guiada por la cobertura que por la calidad de vida tanto de sus educadores, como estudiantes, por supuesto, sin dejar de lado las condiciones acústicas de las aulas para promover espacios de aprendizaje adecuados.

Sin embargo, a ello no sólo debe sumarse esa patología, sino al hecho mismo del tener que subir la voz, en consecuencia aportar a la transformación de la dinámica comunicativa en el aula, en donde sólo se le empezará a prestar atención a un tono de voz fuerte, más del normal, y se abre un espacio al grito como directriz para la disciplina en el aula. Estos eventos fomentan el irrespeto al otro, no por el grito en sí mismo, sino por el hecho de creer de manera in-

la transformación de la dinámica comunicativa en el aula, en donde sólo se le empezará a prestar atención a un tono de voz fuerte, más del normal, y se abre un espacio al grito como directriz para la disciplina en el aula. directa al otro incapaz de hablar de manera natural, en un tono adecuado, sino de enseñarle y promover de manera indirecta que la 
forma de comunicarse sólo es posible cuando se habla por encima del promedio, como si hablar fuerte implicara el tener el mejor argumento frente a cualquier diferencia que surja.

Cuando se está en el aula, si el docente ya ha entrado en el juego del grito, del ruido, a través de su voz, no será posible con la reincidencia de ello lograr la atención de sus estudiantes frente a lo que expone de una manera distinta, puesto que al interior del aula se toma como lo "normal", como lo "natural" lo que se impone, no lo que se dialoga, se transforma el grito y/o el tono de voz fuerte como la única forma reconocida de hablar con el otro, sin que ello sea un proceso consciente.

Le damos al grito o a ese tono de voz fuerte, el poder de transformar lo que pasa o puede pasar en el aula. En consecuencia, se hace referencia al hecho de que para evitar una agresión, el llamado de atención se manifeste con un grito. Así, para el docente es preferible el grito, a que un niño lastime fsicamente a otro. Si miramos su trasfondo, sólo se ha seguido una dinámica que puede haberse gestado en la misma dinámica familiar donde la disciplina se ha aprendido a través del grito y de la voz que se impone, no la que se escucha, para dar paso a la detención abrupta de un comportamiento inadecuado con un llamado a la cordura equívoca, en lugar de promover la cultura del respeto y de la escucha.

Aquí ya hablamos de cómo se legitima una actitud, un comportamiento, una forma de comunicarse, puesto que culturalmente sólo se logra transformar un acto esporádico en un hábito cuando se toma como el parámetro por el cual lograr siempre un mismo resultado, y cómo se incorpora en rutina, y de ahí se hace la transferencia de ese acto a distintos escenarios. Entonces, tendremos el grito como medida preventiva, medida de corrección y medida de negociación sin importar donde se encuentre el docente o se encuentren los niños y las niñas.

Legitimar en este aspecto no es más que convertir un acto, una acción, o un gesto como parte de aquello que nos permite comunicarnos, por lo cual ese grito (enmarcado dentro de lo que hace parte del ruido) se convierte en parte de la comunicación y con ello, se constituye en un elemento natural del acto comunicativo en el aula por el hecho de transformarlo en la forma como logramos la atención del otro, de hacerse "escuchar", pero sobretodo, que otros reconozcan a los docentes.

Al realizar un ejercicio en el aula sobre la contaminación ambiental, un grupo de niños del grado quinto primaria de una institución pública como parte de su clase de Ciencias Naturales, debían identifcar en su colegio aquellos aspectos que generan contaminación y agruparlos según el tipo de contaminación que producían. El ruido, no fue un factor de contaminación visible para ellos. Posteriormente, al hacer el proceso teórico de identifcar dichos factores en la institución y estructurar un mapa mental como resumen de dichos contenidos teóricos expuestos y explicados, se procedió a realizar una serie de entrevistas de manera grupal a los estudiantes de cuarto primaria, para poder observar qué aspectos identifcan ellos como causantes de contaminación ambiental en la institución y dónde el ruido era visible para los niños que moderaban la entrevista, pero era todavía invisible para aquellos entrevistados. Junto a ello, se realizó un sencillo video de sensibilización en el que se enfatizó en el ruido como un factor contaminante en el aula en el 2010. Estos textos audiovisuales fueron observados por niños de diferentes grupos tanto de primaria como de secundaria de la institución en la semana de emprendimiento que organiza la misma. 
Como continuidad a este proceso en el 2011 se realizó con los estudiantes de quinto grado, el proceso de identifcación de los contaminantes ambientales presentes en el colegio. Estudiantes que habían sido entrevistados cuando cursaban cuarto de básica primaria y a quienes se les había presentado los factores contaminantes identifcados en el colegio en el 2010 como cierre de la entrevista grupal. Además, con este grado se realizó el video que se presentó de manera institucional. Este grupo de estudiantes, al igual que el grado quinto en el 2010, no identifcaron el ruido como factor contaminante relevante dentro y fuera del aula.

El ruido, aparecía mencionado a manera de comentario pero inmediatamente ellos mismos expresaban que no era un contaminante como tal, puesto que era normal el ruido. Con este nuevo grupo de estudiantes también se procedió a realizar entrevistas pero de manera individual a cinco personas, de los cuales al menos uno debía ser un docente, quienes también plantean distintos tipos de contaminantes pero no solían hacer referencia al ruido.

Como ejercicio de cierre, se llevó a cabo con este grupo de estudiantes de quinto grado de básica primaria 2011, en lugar de la elaboración de un video, el desarrollo de cinco preguntas. Estas fueron realizadas tres meses posteriores a las entrevistas que ellos mismos realizaban a otros. Durante este receso no se hizo mención de los contaminantes dentro y fuera del aula en la institución.

¿Cree o piensa que el ruido contamina?

¿Por qué considera o piensa que contamina o no?

¿Cómo cree que puede llegar a afectar el ruido en la clase?

¿Por qué es importante hablar en un tono adecuado?
¿Tipos de ruido que identifca dentro y fuera del salón?

Frente a dichas preguntas, sorprende que de treinta niños, once consideren que el ruido no es un contaminante. Al anterior resultado se puede agregar cinco que optaron como respuesta un tal vez, o puede ser, lo cual nos lleva a más de un 50\% entre los que no le darían un lugar defnido al ruido como contaminante.

Además, al hacer referencia a la afectación ocasionada por el ruido en la clase, los seis que incluso contestaron que sí era un contaminante, no logran hacer tangible el efecto que puede producir en el aula. $\mathrm{O}$, mejor aún, a pesar de identifcarlo como contaminante, no relacionan un tono de voz fuerte como un ruido en sí mismo, por lo cual no expresan como ruido el hecho de que un docente levante la voz más de lo normal, pero sí pueden identifcar como ruidos dentro y fuera del salón aquello que cada uno puede producir como los gritos, el hablar con otros, el llorar, el saltar, las risas, la música, los zapateos y los silbidos, entre otros.

Por el contrario, sí relacionan un tono de voz fuerte con el daño que se produce a nivel físico (dolor de cabeza, lesión en los oídos, no lograr escuchar a otros, problemas en la garganta, estrés (...) o cómo afecta la relación con aquella persona (no molestar a la gente, para no asustar, para que no piensen mal, para que no piensen que se gritan, para no irrespetar a los adultos y otros) pero esta afectación es más en función de la relación adulto - niño, no de cómo el ruido puede alterar o puede producir algo en un par, es decir, en cómo puede herir la salud del otro niño o niña, sino en cómo afecta su forma de relacionarse con el docente.

Lo más interesante de este aspecto, es observar cómo en ocasiones se excusa el 
Pero esta afectación es más en función de la relación adulto - niño, no de cómo el ruido puede alterar o puede producir algo en un par, es decirr, en cómo puede herir la salud del otro niño o niña, sino en cómo afecta su forma de relacionarse con el docente. ruido como parte de la naturaleza o identidad de un niño o una niña, en lo natural que es en el aula o en la hora del descanso un tono de voz fuerte, los gritos, las peleas. Cómo por el rol mismo que se tenga en el aula, hablar fuerte se asocia a éste, sobretodo en cómo un tono de voz elevado se ha convertido en la manera de generar un comportamiento o una pausa en el mismo. De lo anterior se vislumbra la transformación del ruido como contaminante a estrategia para la disciplina dentro y fuera del aula.

De acuerdo a esta experiencia se colige que desafortunadamente lo único que se logra es transformar la dinámica comunicativa de la educación, en una forma de relacionarse agresivamente a través de las palabras, por supuesto, según que el tono en el que se dice hace parte del gesto que tiene una palabra y que cuando sobrepasa los límites, además de generar ruido y contaminar el ambiente educativo, obstaculiza los niveles de concentración pues no es posible escuchar claramente a otro y, por supuesto, el proceso de memorización -por ejemplo- no logra generar el insumo para el recuerdo más efectivo de los contenidos del conocimiento que circula en el aula, además de producir un efecto en el otro que no sólo es físico, sino emocional, social y comportamental.

\section{Conclusiones}

A partir de la experiencia con los niños y las niñas del grado quinto, del Colegio Roberto
Garcia Peña, Sede B, jornada de la tarde puede observarse que:

1. El ruido, al interior del aula puede ser ocasionado por la elevación en el tono de voz tanto en estudiantes como en docentes, no sólo por gritos, zapateos, o caída de objetos pesados, pero aún no es visible para estudiantes ni docentes.

2. Es más fácil para los niños ver cómo afecta el ruido su relación con sus docentes pero no con sus compañeros.

3. Hay una conciencia de cómo podría afectarles a ellos mismos pero no a un par.

4. La naturalización del ruido como forma de comunicación inicia y se consolida cuando:

a. Hay una reincidencia en el tiempo. En el diario vivir en el aula lo normal es hablar en tonos de voz fuerte.

b. El grito se convierte en el llamado de atención o la manera en cómo formamos y ponemos límite a la interacción inadecuada en los estudiantes.

c. No se tiene conciencia de la elevación en el tono de voz, se ha automatizado.

d. No se presta atención fácilmente a quien no habla en un tono de voz elevado. Tampoco se llama la atención a quien habla por encima del tono natural de voz.

e. Se legitima el ruido como parte de la manera en que nos comunicamos, sin importar el escenario en el cual nos encontremos.

6. El grito, se convierte en la forma identifcada tanto por estudiantes como docentes para prevenir, corregir, e in- 


\section{cluso dar inicio a la negociación en las situaciones confictivas que se presen- tan dentro y fuera del aula.}

\section{Referencias Bibliográfcas}

Del Valle, E y Almirall, P. (2008). Alteraciones de la voz y condiciones de trabajo en maestros de enseñanza primaria. Aragua, Venezuela. Cuba: Editorial Universitaria. Disponible en: http://site. ebrary.com/lib/bibliotecaustasp/docDetail. action? docID $=10232473 \& \mathrm{p} 00=$ ruido $\% 20$ escuela.

Flores-Domínguez, E. (2009). Contaminación Acústica. Argentina: El Cid editor, Apuntes. Disponible en: http://site.ebrary.com/lib/bibliotecaustasp/docDetail.action? docID = $10327717 \&$ p 00 $=$ contaminaci $\%$ C3 \% B3n \% 20 ac\% C3\% BAstica.

Freirs, C. (2009). Veneno en los oídos. Argentina: El Cid editor, Apuntes. Disponible en: http://site. ebrary.com/lib/bibliotecaustasp/docDetail.act ion? docID $=10317016 \& \mathrm{p} 00=$ contaminaci $\% \mathrm{C} 3 \% \mathrm{~B}$ 3n\%20auditiva.

Preciado, J. (2009). Estudio de la prevalencia de los trastornos de la voz en el personal docente de Logroño: análisis multidimensional de la voz en los profesionales de la enseñanza. España: Instituto de Estudios Riojano. Disponible en: http:/ / site.ebrary. $\mathrm{com} / \mathrm{lib} /$ bibliotecaustasp/docDetail.action?do cID $=10294261 \&$ p00 $=$ estudio $\% 20$ prevalencia $\% 20$ trastornos $\% 20 \mathrm{voz} \% 20$ personal $\% 20$ docente $\% 20$ $\log$ ro $\% 3 \%$ B1o \% 3 A $\% 20$ an $\%$ C3 \% A 11 isis $\% 20$ multidimensional $\% 20 \mathrm{voz} \% 20$ profesionales $\% 20$ ense \%C3\%B1anza.. 
